\title{
Medicine Meets Engineering in Cooperative Design of Collaborative Decision-supportive System
}

\author{
Oscar Frykholm ${ }^{1}$, Ann Lantz ${ }^{1}$, Kristina Groth ${ }^{1,2}$, Åke Walldius ${ }^{1}$ \\ ${ }^{1}$ HCI, CSC, The Royal Institute of Technology, Stockholm, Sweden \\ ${ }^{2}$ CLINTEC, Karolinska Institutet, Stockholm, Sweden \\ \{frykholm,alz,kicki,aakew\}@csc.kth.se
}

\begin{abstract}
Researchers in Human-Computer Interaction have worked together with physicians to specify and create prototypes of a system to be used primarily during multi-disciplinary team meetings. Physicians will use the system to aggregate and present relevant patient information during discussions on diagnosis and treatment, and also to coordinate the cases during the patient care pathway. In this paper we present the cooperative design process and activities conducted within the project. The results are two-fold; we report on the progress of creating the decision-supportive system, and describe how the physicians experience the design process. The design activities have made the physicians reflect on: the lack or loss of patient information, how patient information can be improved, break-downs in their work process, how they learn from each other, and the design methodology itself.
\end{abstract}

\section{Introduction}

ICT development in large, distributed organisations is a difficult task with few approaches to ensure a usable system in the end. In Scandinavia, there is a tradition of early user involvement and collaboration between users and developers, introduced in the 1980's as the Cooperative Design approach (e.g. [1,2]). The involvement of users requires a substantial contribution in time and effort from both parties throughout the design process, but in return it has positive long-term effects on usability and user acceptance of the new system. The approach also tends to break down the barriers between the user and the developer, when they learn about each other's domain.

In cooperative design, the usability of the system itself is usually the main concern, but we argue that by introducing new tools, the work processes and tasks inevitably change too. Therefore, cooperative design affects the users and makes them reflect upon their work, and thereby helping them to continuously refine the requirements of the system throughout the process. In other words, tight coupling between users and developers is necessary in order to create a system that will fit users' needs in the long run.

We report on a project where the users' work processes are highly specialised, where multidisciplinary decision-making to a large degree relies on both personal experiences and scientific research, and where a decision-supportive system has to meet the requirements of a complex set of interdependent work processes. Being researchers in Human-Computer Interaction (HCI), we are confident that we will be able to create new tools and interfaces, but to what extent will the cooperative design process help us engage the physicians who, through their involvement in the process, will have to make sure that the tools enable their new, enhanced work processes?

\section{Background}

The research project Funk-IS was initiated in collaboration with the surgical department (called Gastro) of a university hospital. One of the authors had spent a year working as a post-doc at Gastro when the project application was written in collaboration with the head of Gastro. The main ideas of the project was to make information more easily available during the patient care pathway, to provide better information as a ground for decisions, and that the project should result in usable applications in ten years time.

In our HCI research we have adopted the cooperative design methodology with early user involvement in system and interface design, primarily by using low- and high-tech prototypes. Bødker et al. [2] describe a long-term project which focused on four main activities: 1) mutual learning between the active participants, 2) requirement specification for a system, 3) studying a pilot installation of the system, and 4) dissemination of project findings, especially to the 
users and to the scientific community. The system design, although important, was rather a secondary result in the project. Their main results concern the cooperative design method itself and insights into how technology is developed and introduced in work processes. They also describe the shift of the participants; from being ordinary users to becoming designers and inventors. Participatory design, aka the North-American continuation of cooperative design, is described by Muller \& Kuhn [3] with a set of techniques, such as workshops, mock-ups and prototypes, to be used by designers and users. The two approaches differ due to organisational culture; in Scandinavia researchers collaborated directly with workers and got acceptance from management to stay at the work site, while the North American tradition often gained access at management level without direct connection with the workers (cf. [3, 4]).

Close cooperation with users can also lead to difficulties. Lantz et al. [5] found that the users can become confused by the researchers' roles (a mix of researcher and developer), as well as of the outcome of the project (users want to benefit directly from the results and do not realise that the results and prototypes will not be available as products until 5-10 years).

In accordance with the cooperative design approach, a group of physicians at Gastro is actively working in the project. In all, there are 16 project members from two very different disciplines: nine HCI-researchers (participating from four hours per week to full time), and seven surgeons (participating based on available time besides their clinical work). Our aim has been to get a nicely mixed "dressing for the salad" as the head of Gastro have stated it. In order to have a good dressing the bottle needs to be continuously shaken, which is what we have tried to accomplish using cooperative design methodologies.

Gastro may differ from organisations in general in that it has a long tradition of conducting technology development projects internally with early user involvement. They have for instance developed and implemented technology to be used during multidisciplinary team meetings (MDTMs) between several hospitals within the county council, and several physicians are involved in piloting new telemedicine solutions used in clinical work. This is in line with Coiera [6] who suggests that the medical community "needs to get 'technical' about what we [the medical speciality] mean and about what we want from a design, and we need to work alongside technologists to shape technology, as well as the processes, organisations and cultures within which they will be embedded".

There are about 25 surgeons working at Gastro, which has a close collaboration with several other units within the hospital organisation. Medical care at Gastro is team-based, which in practice means that no physician alone is responsible for a specific patient. A central work process at Gastro is the set of weekly MDTMs, where up to six other hospitals within the region participate using a video-conferencing system and screen sharing. During the meetings the gathered physicians decide in consensus on diagnosis and treatment of the patients, as well as on pre-operative planning and review of post-operative patients. In the project, we have focused on the consensus meetings because they are truly multi-disciplinary; a minimum of three different specialists must be present, at least five physicians must attend, the complete patient case is presented and discussed, and the physicians have to make decisions in consensus. That is, it is probably the most difficult MDTM during the patient care pathway, where a lot of information must be aggregated and presented, and where collaborative and multidisciplinary decision-making is critical.

Decision making in the field is different from decision making in laboratories and, following the underlying ideas of the naturalistic decision theory [7], should be studied in the field: "Experts don't make deliberate $[\ldots]$ decisions but rather know what to do based on prior experiences and domain knowledge" [7, p.1239]. The authors argue that experts exhibit a holistic, intuitive, recognition-based approach to decision-making and problem solving; an expert has a rich conceptual model of the domain, which supports recognising the case into which a specific decision situation fits, and from this recognition retrieves the appropriate solution strategy and applies it with little analysis or reasoning.

Gustavsson et al. [8] report that few clinical decision support systems have reached a sustained clinical acceptance. In order to receive acceptance by the clinicians, the system should for instance be adapted to, and integrated with, new enhanced work processes, and it should be integrated with other systems to avoid manual handling of information. Systems that strengthen rather than replace the clinicians' decisions have a greater chance to get an impact clinically. Niès \& Pelayo found that involving users in the software cycle is a good starting point, but to solve complex usability problems, human factors experts who understand the physicians' work situation should be integrated [9].

To us, these findings confirm the importance of applying a cooperative design approach in which clinicians participate during the whole development process. The key ambition is to adapt the system from the beginning, to design it according to the workflow and the needs and requirements of the users. In this process we are interested in what signs of critical 
distance and reflection regarding current work processes could be assembled from the physicians during our ongoing data collection for requirement specification and design work.

\section{Method}

The Funk-IS project started in September 2008 and as of June 2010, we have conducted a number of parallel activities in the project; field studies (observations and interviews), project meetings, workshops and prototyping sessions. The project is managed from the HCI-side, where also the planning of design activities, analysis of material, and the building of initial prototypes has been carried out. All physicians in the project group have continuously been updated about the work, but they have not participated in every activity. Two of the physicians have taken on a more active role and worked hands-on with creating specifications and evaluating different design proposals. We have also included other physicians (surgeons and radiologists) in evaluations of prototypes and interviews on work processes.

In the beginning of the project, a series of meetings with all project members were held. They consisted mostly of presentations of ongoing work, preliminary results and discussions on goals in the project. The cooperative part of these meetings consisted of, on one hand, the physicians guiding the HCI-researchers in their interpretation of work processes at Gastro, and on the other hand of the HCI-researchers presenting a bird's-eye-view of the work at Gastro. Also early in the project, a number of workshops and demo sessions were conducted, to show-case prototypes developed in other projects, including modifications of the prototypes intended to better suite the physicians' needs. The goal was for the physicians to hands-on test the technology and discuss in what ways it could be used in their work.

Besides earlier long-term fieldwork that has informed the project, two formal rounds of field studies have been conducted. The first field study spanned three weeks in March 2009 and included three pre-operative meetings, and the second field study spanned five weeks in September and October 2009 and included nine consensus meetings. The meetings lasted about one hour each, they were video-recorded and afterwards analysed and partly transcribed. We have also observed physicians in the conduct of their work, for instance by shadowing senior surgeons during all steps of the patient care pathway. Formal interviews have been done with surgeons and radiologists, and informal conversations have been held with all surgeons at Gastro as well as with radiologists and an oncologist. Our orientation to field research follows the approach taken by Hughes et al. [10], emphasising the description of observable features of the work.

Following initial fieldwork, when project members cooperatively had developed high-level specifications of the intended system, prototyping sessions were conducted to start designing the system interface. During two three-day sessions, one of the physicians teamed up at the HCI department together with three to four researchers in hands-on, cooperative design activities and produced sketches of the system. In all, the project meetings, fieldwork, and prototyping sessions represent a long-term collaboration with Gastro that has helped develop a trust between researchers and surgeons. Such investment in relationships among project members is critically important.

\section{Results}

The surgeons explicitly stated in the beginning of the project that they required tools to quickly process information during multi-disciplinary team meetings. The need for tools has also been reported in [11] where it has been suggested that physicians must change from "being memory repositories to information managers".

Apart from introducing new technology and thereby influencing the physicians' work, we did not set out in the beginning to deliberately influence their work in any specific direction. As the cooperative design work proceeded, we have learned that the design process has influenced the physicians in the way they see their work and made them reflect on how to improve certain processes. We can therefore present results not only on the development of a decision-supportive tool, but also - and perhaps of more interest - on how the physicians have responded to the design process.

\subsection{Interaction design}

The main work at Gastro obviously concerns surgical treatment of patients, an area that we as HCIresearchers do not intend to improve. What we on the other hand are interested in, and where we believe we make a difference, is in the way physicians manage patient information. We subsequently set out to learn how patient information is used and accessed at Gastro in current work processes. Results in this section are primarily based on field studies, but have been verified in workshops and interviews with physicians.

First of all, we learned that patient information is not used in isolated situations. There is a structured process at Gastro that patients follow, the patient care pathway, which consists of four main steps:

- Coordination: Review of patient information to 
ensure it is sufficient for continued assessment.

- Decision (MDTMs): All relevant patient information is discussed at a consensus meeting, where decision on diagnosis and treatment is reached. The case is reviewed at a preoperative meeting, if surgery is needed.

- Treatment: Before, during and after surgery, surgeons access patient information in order to plan the surgery and write post-surgical notes.

- Post-operative: A few months after surgery, patient outcome is discussed at an MDTM, in order to present feedback on the treatment.

Patient information is accessed and complemented during the whole patient care pathway, by several physicians and in different situations. In the end, they have produced a full description of the case, including relevant examinations, diagnosis, treatment and outcome. This means that specific information a physician might be interested in (such as outcome of similar cases during consensus meetings) is produced and used asynchronously; they document actions and patient information not only to know what they have done, but also for future use.

A consensus meeting lasts 60-90 minutes, handles ten cases and is attended by five to twenty physicians. As all physicians are not located in the same hospital, video-conferencing and screen sharing are used. The different roles of the participants, the workflow of the meeting and the way information is presented, make it possible for all participants to follow a clear structure of information exchange $[12,13]$.

During the consensus meetings, advanced technology is used, such as radiology software and screen sharing. But when it comes to presenting and searching for patient information, physicians must rely on their own memory of the cases or printouts of the patient record that they have brought with them. As they are not always able to answer specific questions, the meetings become vulnerable as to who are present and what they remember. Radiology examinations are an important part of the meetings today, as they are the best representation of the patient. Textual information is equally important but not utilised to the same extent; hence we believe that easier access to patient information, as well as the inter-play of text and images will add new dimensions to the meetings.

Early in the project the physicians explained that access to medical information was cumbersome and contained too much "noise", i.e. it takes time to find specific information when needed. We consequently started working on structuring and categorising the medical information, based on what is actually relevant during the consensus meetings. We have made several iterations of this exercise, and the list now more or less constitutes a complete profile of the patient.
Another early observation was that the physicians lacked a tool for visualising, and collaboratively working with, medical information during the consensus meetings. To initiate the discussion on what such a tool could look like and what support it would add to the meeting, we presented a first conceptual idea of a "collaborative clinical workspace" [14]. At this point we experienced the difficulties of working in two different domains, as only a conceptual idea of a tool was very difficult to grasp for the physicians. We then proceeded to make high-level sketches of what such a system could look like and presented them in workshops, followed by interviews and evaluations with the physicians. The immediate result was that the physicians actually understood what we were talking about and could discuss how it could be used in their work. During the two prototyping sessions the sketches and requirements have been further refined. The sketches are now being realised into high-fidelity prototypes, and will be evaluated together with the physicians in order to study what positive and negative effects the addition of collaborative tools bring to the meetings, and how they affect the discussions.

\subsection{User responses to design initiatives}

Examples used in this section are mainly taken from two workshops and one prototyping session. The first workshop was conducted quite early in the project, and the focus was on structuring all the patient information that is relevant during consensus meetings. The second workshop was held a year later and now included patient information that is used during the whole patient care pathway. Three physicians participated in each workshop, but only one of them in both of the workshops. The prototyping session was also conducted later in the project, with one physician during three days. In this session, we focused on detailed comparison of patient information for a few specific patients, and also on creating a design proposal for the intended decision-supportive system.

In the design activities where the flow of patient information between different physicians was discussed, the participating physicians have on a number of occasions expressed insight into lack, or loss, of information. For example, in one workshop, questions were raised about how information from one step to another in the patient care pathway was transferred. They recognised that there were situations where information sometimes was left behind or even dropped out of the system. In another exercise, all available information on a specific patient (including all notes in the patient record along with transcripts of the MDTMs where the patient had been discussed) had been gathered and laid out on a large table. One of the 
physicians noticed that the discussion on one possible treatment during the consensus meeting, which had been unanimously discarded, was not recorded in the decision. They had discussed why the particular treatment should be avoided in this case, but still at least two physicians (who had not attended the meeting) later on had discussed the treatment option with the patient.

The physicians also found situations where they could improve and extend patient information. For instance, involving the patient herself could be advantageous both for the physicians and the patients:

Surgeon1 The patient needs to do more things themselves!

Manager The patient can see, they would like to know where they are [in the process].

Another example is when Surgeon2 sees the possibility to make patients contribute with specific information to the system: "This is really great, don't have to call the patient because the information is included [i.e. the patient can fill out a form in preparation for a meeting with the physician]".

At one occasion during the workshop on the patient care pathway, one of the managers realised that they might even have situations causing serious breakdowns in their process:

Manager What is the communication between these two steps today?

Surgeon2 It doesn't exist.

Manager Then there is a great need [to improve it].

Managers and physicians all have a common understanding of how the patient care pathway should be practised, but in reality, how the different steps actually are conducted depends on individual physicians and on the practical situations of the work. The physicians continuously improve the pathway in discussions during for instance internal team meetings. But during the cooperative design activities other flaws in the process could be identified more systematically.

The different design activities have also brought the physicians together and offered them time to discuss how their work is performed today and what can be improved. It has enabled them to learn from each other's work and reflect on how things actually work (or not). In one of the exercises, a transcript of the discussion during a consensus meeting is presented to the physicians. They seem somewhat dissatisfied with what they read, and Surgeon3 even comments: "Why all these words when what we are looking for is something very exact?". During the discussions between the physicians, they gain insight in each other's way of doing different work routines, they learn from each other, and explicitly express the need for a more systematic way of working. To a certain extent, they calibrate their work, and ponder over existing needs and what information is desired in what way. Thus, reflections on how to increase the quality of their work are an essential part of the discussions.

As a final remark, we have noticed that the physicians reflected on the design methodology used in the project. In the prototyping session, Surgeon1 spontaneously commented: "This is a brilliant way of working with development. [] Our patient care pathway makes us light-years ahead. It feels great since we are so far ahead. [] It's really bracing.". After the workshop on the patient care pathway, the manager who had participated discussed with the head of Gastro about conducting a similar workshop with all of the staff at Gastro. Apparently they realised that some of the activities of cooperative design can also be applied to their organisational development work in the surgical department. Hence, our methods in the project have improved the quality of their work processes, and via design activities and discussions the physicians are forced to take a holistic perspective on their work.

\section{Discussion}

In the Funk-Is project we have as HCI-researchers worked for nearly two years together with physicians in the design of a decision-supportive system, which is intended to help physicians manage the vast amount of information encountered in their daily work. It has been a necessity for us to conduct much of the work at the hospital, and to invest time in getting to know the physicians, the department and their work processes, whereby we have reached a detailed level of understanding about the users and their context. We have used and presented this knowledge in the design activities, which have both secured user acceptance, and also helped the physicians to gain a holistic view of their work [8]. The design activities, as well as the continuous presence of HCI-researchers at the hospital, have inevitably affected the physicians and the way they see their work processes. In other words, technology development has lead to development of the work processes, a critical effect that always has to be taken into account in cooperative design projects. We think it is fair to conclude that the cooperative design process has helped us to engage the physicians in critical assessments of their current work processes, thereby securing that the new tools will become well adapted to user needs. The kind of responses we have received - on accessibility and quality of patient information, on process enhancements and organisational re-design - make us confident that the approach can help us avoid the danger of having technology, rather than professional know-how and 
commitment, drive the design process. We also believe that Gastro, being an organisation where internal development projects are common, have realised that they need to work close with "technologists" to improve their technology and work processes (cf. [6]).

On one hand, the project has created commitment and interest among the physicians, and the results of the project are promising. On the other hand, conducting a research project, or for that matter any technology development project, within a work place requires a substantial amount of time from the users. The physicians are extremely busy, and work long hours even without involvement in the project. We have therefore been forced to adapt to their schedule and plan the number and format of the activities according to their availability. It has partly been solved by a few physicians devoting research time, which is included in their working hours, to the project. Because of these constraints, and although the project has focused on the cooperative design method, we can also conclude that the HCI-researchers have become more familiar with the physicians' domain, than vice versa. With one exception; the senior surgeon who has participated in most of the workshops and the two prototyping sessions, has been able to generate sophisticated interaction design ideas and to some extent act within the field of HCI [2].

Working together with users is a necessity, but one should not forget that people skilled in HCI are professionals with a set of tools and knowledge grounded in a certain design methodology. Users are guided by the HCI professionals, who know "whereto" the project should go and "why", and who can reflect on the progress of interaction design [14]. With Gastro's history of user involvement in their development projects, they are comfortable with using the cooperative design method. User involvement is natural for them, but they have previously not had the knowledge of what design activities to use. Furthermore, as the initiative to this project was partly taken from the head of Gastro, management involvement comes naturally and we fulfil the requirements of both cooperative and participatory design $[1,4]$. All in all, the long-term goal of a project like this is actually to increase the collaboration between different disciplines; medicine, engineering and research. The "dressing for the salad" is not only a nice expression, it has become the preferred way of working, in order to make sure that state-of-the-art medicine is continuously improved using state-of-theart technology.

\section{Acknowledgements}

This work was supported by VINNOVA. We thank all the members of Gastro who have cooperated and participated in this study.

\section{References}

[1] J. Greenbaum and M. Kyng, "Design at work: cooperative design of computer systems", Lawrence Erlbaum Associates, Inc., Mahwah, NJ, 1992.

[2] S. Bødker, P. Ehn, D. Sjögren, and Y. Sundblad, "Cooperative Design - perspectives on 20 years with 'the Scandinavian IT Design Model'", Proceedings of NordiCHI 2000, Stockholm, Sweden.

[3] M.J. Muller and S. Kuhn, "Participatory Design", Communications of the ACM, 36 (6), 1993, pp. 24-28.

[4] D. Schuler, and A. Namioka, "Participatory design: Principles and practices", Lawrence Erlbaum Associates, Hillsdale, NJ, 1993.

[5] A. Lantz, M. Räsänen, and P-A. Forstorp, "Co-operative design projects", In J. Bornebusch and P. Hernwall (eds.), No.1:2006, Vol.1, 30-41, M3, Södertörns högskola, Tallin:Printon Trükikoda AS, 2006.

[6] E. Coiera, "Putting the technical back into socio-technical systems research", International Journal of Medical Informatics 76, S1, 2007, pp. S98-S103.

[7] W.W. Zackary, and J.M. Ryder, "Decision support systems: Integrating decision aiding and decision training", Handbook of Human-Computer Interaction. M. Hellander, T.K. Landauer, P. Prabhu (eds.), 1997, pp.1235-1258.

[8] J. Gustavsson, U. Fors, and J. Ellenius, "Analys av förekomst och behov av medicinska beslutsstödssystem i Sverige", Technical report, Department of learning, informatics, management and ethics, LIME, 2007.

[9] J. Nies and Sylvia Pelayo, "From users involvement to users' needs understanding: A case study". International Journal of Medical Informatics, 79 (4), Human Factors Engineering for Healthcare Applications Special Issue, April 2010, pp. e76-e82.

[10] J. A. Hughes, D. Randall, and D. Shapiro, "Faltering from Ethnography to Design”. Proceedings of CSCW 1992.

[11] J. Collins, "Evidence-Based Medicine", Journal of the American College of Radiology, 4 (8), 2007, pp. 551-554.

[12] K. Groth, O. Frykholm, R. Segersvärd, B. Isaksson, and J. Permert. "Efficiency in Treatment Discussions: A Field Study of Time Related Aspects in Multi-Disciplinary Team Meetings", Proceedings of CBMS, IEEE, 2009.

[13] O. Frykholm, and K. Groth, "Shared visualization of patient information", Position paper, Workshop on Interactive Systems in Healthcare (WISH), ACM CHI 2010.

[14] S. Bødker, and O. S. Iversen, "Staging a professional participatory design practice: moving PD beyond the initial fascination of user involvement". Proceedings of NordiCHI 2002, Aarhus, Denmark. 